\title{
Implementing Neural Network and Multi resolution analysis in EEG signal for early detection of epilepsy
}

\author{
Sachin Shrestha*, Rupesh Dahi Shrestha ${ }^{+}$, Bhojraj Thapa ${ }^{* *}$ \\ Department of Electronics and Communication Engineering \\ Nepal Engineering College, Changunarayan, Bhaktapur, Nepal
}

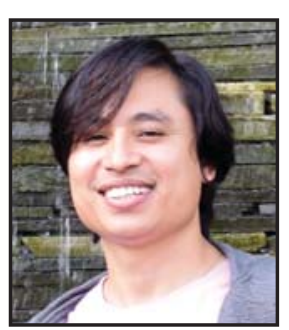

Sachin Shrestha has completed his Masters in Information and Communication Engineering from IOE, Pulchowk Campus. He has been involved in teaching profession for the last 11 years and is currently working as Assistant Professor at Nepal Engineering College, Changunarayan, Nepal. His research interests are ultra-low powered system design, biomedical signal processing, embedded system design and image processing.

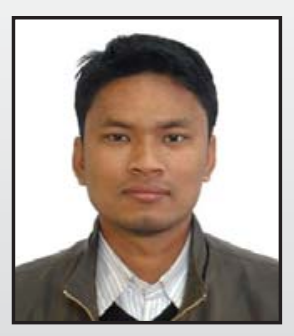

Rupesh Dahi Shrestha received the $B E$ degree in Electronics and Communication from Nepal Engineering College in 2005, and MSc degree in Information and Communication Engineering from Pulchowk Campus in 2013. He has experience of 13 years in teaching and is currently working as an Assistant Professor and the Head of the Department of Electronics and Communication Engineering, Nepal Engineering College. His research interests include signal processing, robotics and embedded system.

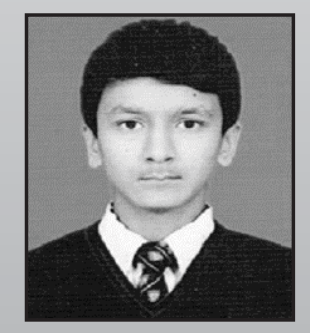

Bhoj Raj Thapa is an undergraduate student at Nepal Engineering College, Pokhara University. He has completed +2 from Liverpool International College under HSEB. He has done projects in his college life related to Android App Development, Signal Processing, and Embedded Systems.

\begin{abstract}
Epilepsy is a neurological disorder of brain and the electroencephalogram (EEG) signals are commonly used to detect the epileptic seizures, the result of abnormal electrical activity in the brain. This paper focuses on the analysis of EEG signal to detect the presence of the epileptic seizure prior to its occurrence. The result could aid the individual in the initiation of delay sensitive diagnostic, therapeutic and alerting procedures. The methodology involves the multiresolution analysis (MRA) of the EEG signals of epileptic patient. MRA is carried out using discrete wavelet transform with daubechies 8 as the mother wavelet. For EEG data, the database of MIT-BIH of seven patients with different cases of epileptic seizure was used. The result with one of the patients showed presence of a unique pattern during the spectral analysis of the signal over different bands. Hence, based on the first patient, similar region is selected with the other patients and the multi-resolution analysis along with the principal component analysis (PCA) for the dimension reduction is carried out. Finally, these are treated with neural network to perform the classification of the signal either the epilepsy is occurring or not. The final results showed 100\% accuracy with the detection with the neural network however it uses a large amount of data for analysis. Thus, the same was tested with dimension reduction using PCA which reduced the average accuracy to $89.51 \%$. All the results have been simulated within the Matlab environment.
\end{abstract}

Keywords : Electroencephalography, Epileptic seizure, Discrete wavelet transform, Daubechies 8, PCA, Neural Network. 


\section{INTRODUCTION}

Electroencephalography (EEG) is the recording of electrical activity along the scalp. It measures voltage fluctuations resulting from ionic current flow within the neurons of the brain. In clinical context, it refers to the recording of the brain's spontaneous electrical activity over a short period of time, usually 20-40 minutes, as recorded from multiple electrodes placed on the scalp [1]. The diagnostic applications generally focus on the spectral content of EEG, that is, the type of neural oscillations that can be observed in EEG signals.

The EEG signal contains several spectral components. The amplitude of a human surface EEG signal ranges from 10 to $100 \mu \mathrm{V}$ while the important frequencies from the physiological viewpoint lie in the range of 0.1 to 30 Hz. The standard EEG clinical bands are categorized as delta ( 0.1 to $3.5 \mathrm{~Hz}$ ), theta (4 to $7.5 \mathrm{~Hz}$ ), alpha (8 to $13 \mathrm{~Hz}$ ), and beta (14 to $30 \mathrm{~Hz}$ ) bands [2]. EEG signals with frequencies greater than $30 \mathrm{~Hz}$ are categorized as gamma waves.

Epilepsy is a chronic neuronal disorder or group of disorders, characterized by the recurrent (two or more) seizures that usually recur unpredictably in the absence of provoking factors. It is linked to an abnormal and excessive discharge from a set of neurons in a specific locus of the brain. This clinical manifestation consists of sudden and transitory abnormal phenomena which may include alterations in consciousness, motor, sensory, autonomic or psychic behaviours. It affects almost all groups of the society. The prevalence and incidence of epilepsy is varying among the countries with heterogeneous figures.

\section{A. Motivation}

About $1 \%$ of people worldwide (65 million) are suffering from epilepsy, [3] and nearly $80 \%$ of cases are in developing countries [4]. Epilepsy becomes more common with the growing age of people [5]. In the developed world, onset of new cases occurs most frequently in infants and the elderly [6] while in the developing world this is in older children and young adults [7], due to differences in the frequency of the underlying causes. It has been observed that about $5-10 \%$ of all people will have an unprovoked seizure by the age of 80 [8], and the chance of experiencing a second seizure is between 40 and 50\% [9]. Epilepsy cannot be cured, but seizures are controllable with medication in about $70 \%$ of cases [10]. In those whose seizures do not respond to medication, surgery, neurostimulation or dietary changes may be considered. Not all cases of epilepsy are lifelong, and a substantial number of people improve to the point that medication is no longer needed.

Automated seizure onset detection will enable the engineering of novel therapeutic and alerting systems to ease the burden of such seizures. There might be therapeutic system capable of detecting and reacting to the onset of a seizure, a local electrical, thermal or neurochemical stimulus that can halt the progression of a seizure prior to the development of clinical symptoms. Moreover, just-in-time, local therapy could relieve patients of the toxic side effects that accompany systemic administration of multi anti-epileptic drugs. An alerting system equipped with seizure onset detection could warn the patient of seizure prior to the development of the debilitating symptoms, or could notify a family member so that the consequences of a seizure can be limited.

\section{B. Onset detection of epileptic seizure}

Various states of art designs have been implemented to analyse EEG signals. EEG signals have been used widely to analyse not just the disorders associated with the brain but also with the various states of the brain like the emotions associated with the nervous systems. Research into seizure detection methods began with the development of seizure event detectors [11], the difference between them being the increased accuracy performance with the later. The detectors developed were meant to detect the seizures of any individual with epilepsy i.e. they were patient non-specific. The variability within EEG severely limited the detection accuracy of such detectors. To improve the performance, investigators developed patient specific event detectors i.e. detectors that could be tailored to EEG of an individual [12]. Years later, the development of diagnostic and therapeutic applications that require initiation following seizure onset motivated the development of seizure onset detection algorithms.

One of the earliest patient non-specific seizure event detectors was the one developed by Gotman [11] in 1982. The Gotman algorithm searches 
for the Hallmark sign of seizures i.e. rhythmic activity. The Reveal algorithm developed by Wilson [13] decomposes two second EEG epochs from each input channel into time-frequency atoms using the Matching Pursuit algorithm. Hand coded and neural network rules are then employed to determine whether features derived from the atoms of a channel are consistent with a seizure taking place on that channel. Saab developed a patient non-specific seizure onset detector [14] algorithm that uses features derived from a wavelet decomposition of each EEG channel to estimate the probability of a seizure event. Qu developed the first patient specific seizure onset detection algorithm [15][16][17][18]. Shoeb had proposed the machine learning based patient specific seizure onset detector [19]. The detector uses a support vector machine to classify a feature vector that automatically encodes the time evolution of spectral and spatial features within the scalp EEG. Sriram Ramgopal et al summarized the works done so far in the onset detection using different approaches [20]. Current trends are more inclined toward the supervised learning techniques however it is restricted to the epilepsy detection [21].

A lot of works has been done on the onset detection of the epilepsy however they are confined to the detection of the epilepsy rather than the effort towards the predictive model for the occurrence of the epilepsy. This paper is focussed more on the feature analysis for the earlier detection of the possible occurrence of epilepsy.

\section{ALGORITHM DEVELOPMENT}

The overall algorithm comprises of basically two major sections and is basically the continuation of the research work published in the September issue of SCITECH Nepal [27]. The first one is the extraction of the signal, its pre-processing and multi-resolution analysis of the signal and the second one is the classification based on extracted features using Neural Network and PCA. The research work is basically the continuation of the work being published in September 2018 issue of SCITECH Nepal [29].

\section{A. EEG signal extraction and pre-processing}

For this research paper, the database of MIT BIH has been used. The different cases presented in the database have been grouped into 23 cases, collected from 22 subjects (5 males, ages 3-22; and 17 females, ages 1.5-19) [19][22][23][24]. Instead of using data of all the patients, only those were selected who has effective number of seizure patterns and that they aren't close enough. Also, the seizures should occur within 1 hour. Based on this, only 7 patients were selected for the analysis. Here in this paper only one patient out of them has been chosen as specific case for the study purpose.

The technical details associated with the signal include the usage of sampling frequency of $256 \mathrm{~Hz}$, sampling period of 0.00390626 second, duration of 1 hour for most of the data and the 23 leads for the analysis of each case.

\section{B. Discrete wavelet transform of the EEG signal}

The Discrete Wavelet Transform analyses the signal at different frequency bands with different resolutions by decomposing the signal into a coarse approximation and detail information. DWT employs two sets of functions, called scaling functions and wavelet functions, which are associated with low pass and high pass filters, respectively. The decomposition of the signal into different frequency bands is simply obtained by successive high pass and low pass filtering of the time domain signal.

The original signal $\mathrm{x}[\mathrm{n}]$ is first passed through a half band high pass filter $\mathrm{g}[\mathrm{n}]$ and a low pass filter $\mathrm{h}[\mathrm{n}]$. After the filtering, half of the samples can be eliminated according to the Nyquist rule, since the signal now has a highest frequency of $\mathrm{pi} / 2$ radians instead of pi, where 2pi is the sampling frequency, fs in terms of radian. The signal can therefore be subsampled by 2 , simply by discarding every other sample. This constitutes one level of decomposition and can mathematically be expressed as follows:

$$
\begin{aligned}
& \mathrm{Y}_{\text {high }=} g[k]=\sum_{n=0} x[n] \cdot g[2 k-n] \ldots \ldots(1) \\
& \mathrm{Y}_{\text {low }}=h[k]=\sum_{n=0} x[n] . h[2 k-n] \ldots \ldots .(2)
\end{aligned}
$$

where, $\mathrm{Y}_{\text {high }}[k]$ and $\mathrm{Y}_{\text {low }}[k]$ are the outputs of the high pass and low pass filters, respectively, after subsampling by 2 .

This decomposition halves the time resolution since only half the number of samples now characterizes 
the entire signal. However, this operation doubles the frequency resolution, since the frequency band of the signal now spans only half the previous frequency band, effectively reducing the uncertainty in the frequency by half. The above procedure, which is also known as the sub-band coding, can be repeated for further decomposition. At every level, the filtering and subsampling will result in half the number of samples (and hence half the time resolution) and half the frequency band spanned (and hence double the frequency resolution).

With the subsampling operator $\downarrow$ (down-sampling), shown in equation (3), equations (1) and (2) can be written more concisely, shown as equations (4) and (5).

$$
\begin{aligned}
& (\mathrm{Y} \downarrow n)[k]=\mathrm{Y}[k n] \\
& \mathrm{Y}_{\text {low }}=(x * g) \downarrow 2 \\
& \mathrm{Y}_{\text {high }}=(x * h) \downarrow 2
\end{aligned}
$$

\section{Calculation of energy of different bands of EEG}

DWT decomposes original signal into two different frequency bands in its $1^{\text {st }}$ level decomposition. In this research work, the decomposition has been done up to five levels resulting the six different frequency bands. The so obtained frequency bands are:

TABLE I

Frequency bands and associated range of frequency

\begin{tabular}{|c|c|}
\hline Frequency bands & Range of frequency (Hz) \\
\hline Band 1 & 0 to 4 \\
\hline Band 2 & 4 to 8 \\
\hline Band 3 & 8 to 16 \\
\hline Band 4 & 16 to 32 \\
\hline Band 5 & 32 to 64 \\
\hline Band 6 & 64 to 128 \\
\hline
\end{tabular}

After obtaining the coefficients for these different frequency bands, the energy associated with each of them is calculated using the formulation based on Parseval's theorem as shown in equation (6).

$$
\begin{aligned}
& E D i=\sum_{j=1}^{N}\left|D_{i j}\right|^{2}, i=1, \ldots \ldots l \ldots \ldots \ldots(6) \\
& E A_{l}=\sum_{j=1}^{N}\left|A_{i j}\right|^{2} \ldots \ldots \ldots \ldots \ldots \ldots \ldots \ldots
\end{aligned}
$$

where $i=1, \ldots \ldots, l$ is the wavelet decomposition level from level 1 to level $l . \mathrm{N}$ is the number of the coefficients of detail or approximate at each decomposition level. $E D_{i}$ is the energy of the detail at decomposition level $i$ and $E A_{l}$ is the energy of the approximate at decomposition level $l$. The value of $l$ is taken 5 . Since the number of coefficients vary with the level, the values obtained have been scaled down by the number of coefficients.

In DWT, it is very important to choose a suitable wavelet alongside the number of levels of decomposition. Here, the Daubechies 8 has been chosen as a mother wavelet for DWT. The reason behind choosing this wavelet for EEG analysis is its smoothing feature which is suitable for detecting changes of the EEG signals. The scaling function and the wavelet function of this mother wavelet are shown in Fig. 1 and Fig. 2. These wavelets are asymmetric in nature and shows bi-orthogonal nature. This wavelet analysis can represent EEG sub-bands as a weighted sum of shifted and scaled version of an original wavelet without losing any information of energy. Since this transformation can retain the frequency as well time properties of the signal, this is considered superior to the other methods like Fourier transform for this research work.

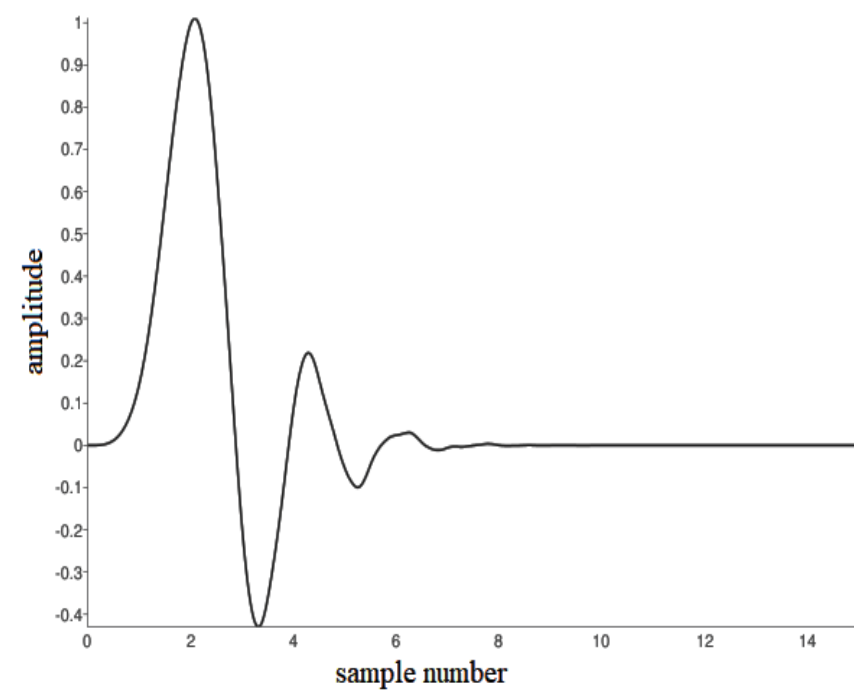

Fig. 1 Scaling function of Daubechies 8 wavelet 


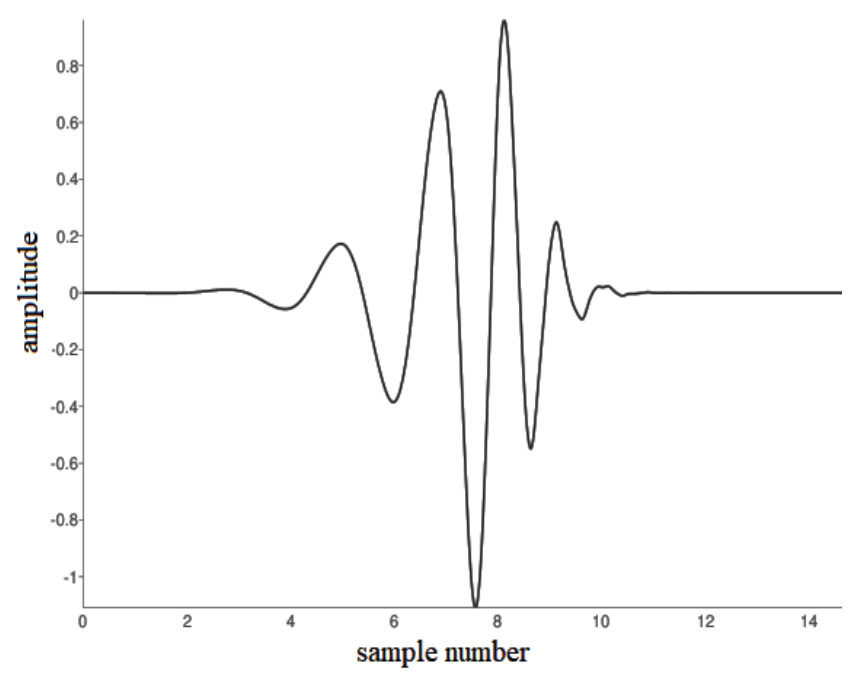

Fig. 2 Wavelet function of Daubechies 8 wavelet

\section{Windowing and shifting for energy calculation}

For the calculation of the energy of different bands, the calculation is done over a window of 256 samples which corresponds to the duration of 1 second. Next, the window is shifted by 128 samples that corresponds to the duration of 0.5 second. The window so taken is of rectangular nature and this window based calculation helps in obtaining better resolution for time. Next, the shift by $50 \%$ i.e. 128 samples with window size of 256 samples will help in eliminating the edge effect of the window. Thus the period of 1 hour i.e. 3600 seconds will give $6 \times 7199$ values corresponding to the each lead of EEG signal.

\section{E. Neural Network and PCA}

After the feature extraction process of the EEG signal extracted from CHB-MIT database, the most prior mechanism is to interpret those features and selection of the features. The extracted features are then analysed and classified on the basis of supervised learning classification technique. To extract the features using NN, energy values of the EEG signals was reshaped into a two dimensional matrix to create an input matrix. Then the machine was trained as per features to detect the unknown EEG signal if that is epileptic or non-epileptic. So, training and testing will be summarized process of the research work which will be done using Neural Network Toolbox of MATLAB.
The feedforward network was developed for the purpose that can be trained to classify inputs according to target classes. The network training function of the neural network updated weight and bias values according to the scaled conjugate gradient method. Backpropagation was used to calculate derivatives of performance with respect to the weight and bias variables [25]. The row vector of the hidden layer is sized as 50. Cross-entropy was used as performance calculation method. It calculates a network performance given targets and outputs, with optional performance weights and other parameters. The function returns a result that heavily penalizes outputs that are extremely inaccurate, with very little penalty for fairly correct classifications. Minimizing cross-entropy leads to good classifiers. The targets were divided into three sets using random indices as training, validation, and testing and the weightage of each was taken as $70 \%, 15 \%$ and $15 \%$ respectively.

Principal component analysis (PCA) is a statistical procedure that uses an orthogonal transformation to convert a set of observations of possibly correlated variables into a set of values of linearly uncorrelated variables called principal components. The PCA has been chosen here to check if the reduced dimension, here it has been reduced to 24 , can give the equally effective results. Here the implementation of PCA has centred the data and used singular value decomposition algorithm [26].

\section{RESULT AND DISCUSSION}

The result of MRA with one of the patients has already been published [27]. From the result of MRA of that patient, the conclusion was made that only 3 of the bands were more effective and hence only last three bands were used as features for the analysis. Next, the usage of only few of the leads instead of all could also generate equally effective results. Hence, only 8 different leads were used. The choice of the leads was made based on the fact that the data of all the patients didn't consist of data of all the leads in similar fashion. Hence, only those that could give us the effective similarity were used. This led to the choice of 8 leads. Instead of using all the patients, only those were selected who had effective number of seizure patterns and that they aren't close enough and were within 1 hour. Based on this, only 7 patients were selected for analysis. 
TABLE II

Info of patients being used for analysis

\begin{tabular}{|c|c|c|}
\hline Patient No & Gender & Age(Years) \\
\hline 1 & Female & 11 \\
\hline 3 & Female & 14 \\
\hline 5 & Female & 7 \\
\hline 8 & Male & 3.5 \\
\hline 14 & Female & 9 \\
\hline 15 & Male & 16 \\
\hline 18 & Female & 19 \\
\hline
\end{tabular}

For the inputs to the neural network, and basically for the early onset detection of epilepsy, MRA results of EEG signals after windowing, of $1400^{\text {th }}$ second prior to seizure till $400^{\text {th }}$ second prior to seizure are taken. This forms the matrix input of [480000 x N], where $\mathrm{N}$ is the number of files present for each patient. Since, this could lead to need of larger input, this has been reduced to 24 dimension using PCA and the $\mathrm{NN}$ is further implemented.

\section{A. Evaluation procedure}

For the analysis of the result, positive predictive value (PPV), negative predictive value (NPV) and accuracy were calculated. The positive predictive value (PPV) is defined as the ratio of number of true positive (TP) by the summation of number of true positive and number of false positive (FP). A "true positive" is the event that the test makes a positive prediction, and the subject has a positive result under the gold standard, and a "false positive" is the event that the test makes a positive prediction, and the subject has a negative result under the gold standard. Likewise, the negative predictive value (NPV) is defined as the ratio of number of true negative (TN) by the summation of number of true negative and number of false negative (FN). A "true negative" is the event that the test makes a negative prediction, and the subject has a negative result under the gold standard, and a "false negative" is the event that the test makes a negative prediction, and the subject has a positive result under the gold standard. The accuracy is calculated as the ratio of summation of true positive and true negative to the summation of true positive, true negative, false positive and false negative [29]. All these could be presented well by the confusion matrix which is also known as error matrix and is widely popular to analyse problems in the field of machine learning and specifically the problem of statistical classification [28].

The confusion matrix for one of the patients after $\mathrm{NN}$ is shown in Fig. 3.

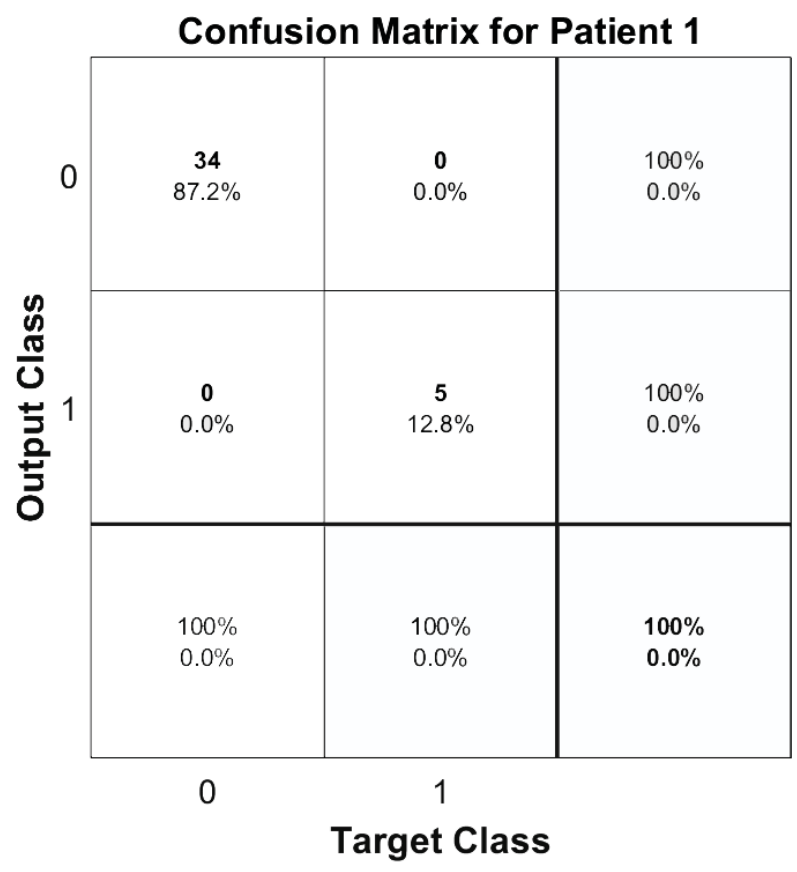

Fig. 3 Confusion matrix for Patient 1 without PCA

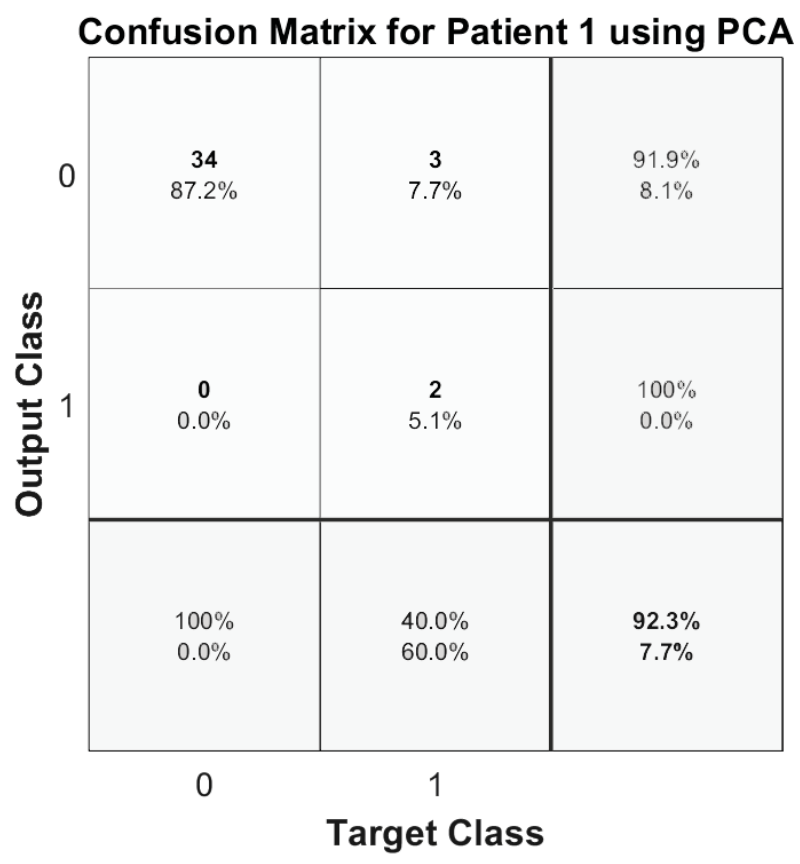

Fig. 4 Confusion matrix for Patient 1 with PCA 
The summarized results for all the patients after using and not using PCA as depicted by the confusion matrix as shown in Fig 3 and Fig 4 is presented in the Table II and Table III.

TABLE III

Summarized results for all the patients without using pca

\begin{tabular}{|c|c|c|c|c|c|c|c|c|c|}
\hline Patient & TN & FP & Sp & FN & TP & Se & FDR & NPV & Accuracy \\
\hline 1 & 34 & 0 & 100 & 0 & 5 & 100 & 100 & 100 & 100 \\
\hline 3 & 30 & 0 & 100 & 0 & 4 & 100 & 100 & 100 & 100 \\
\hline 5 & 34 & 0 & 100 & 0 & 3 & 100 & 100 & 100 & 100 \\
\hline 8 & 14 & 0 & 100 & 0 & 5 & 100 & 100 & 100 & 100 \\
\hline 14 & 17 & 0 & 100 & 0 & 6 & 100 & 100 & 100 & 100 \\
\hline 15 & 26 & 0 & 100 & 0 & 9 & 100 & 100 & 100 & 100 \\
\hline 18 & 28 & 0 & 100 & 0 & 4 & 100 & 100 & 100 & 100 \\
\hline
\end{tabular}

TABLE IV

Summarized results for all the patients using pca

\begin{tabular}{|c|c|c|c|c|c|c|c|c|c|}
\hline Patient & TN & FP & Sp & FN & TP & Se & FDR & NPV & Accuracy \\
\hline 1 & 33 & 5 & 86.8 & 1 & 0 & 0 & 97.1 & 0 & 84.6 \\
\hline 3 & 30 & 0 & 100 & 0 & 4 & 100 & 100 & 100 & 100 \\
\hline 5 & 34 & 2 & 94.4 & 0 & 1 & 100 & 100 & 33.3 & 94.6 \\
\hline 8 & 15 & 2 & 87.5 & 0 & 3 & 100 & 100 & 60 & 89.5 \\
\hline 14 & 16 & 2 & 88.9 & 1 & 4 & 80 & 94.1 & 66.7 & 87 \\
\hline 15 & 24 & 6 & 80 & 2 & 3 & 60 & 92.3 & 33.3 & 77.1 \\
\hline 18 & 26 & 0 & 100 & 2 & 4 & 66.7 & 92.9 & 100 & 93.8 \\
\hline
\end{tabular}

From tables II and III, it can be concluded that the one without using Neural Network gives better result and in fact gave $100 \%$ accuracy however at the same time needs larger amount of data for analysis. With PCA implemented which could lead to lesser data for processing, the processing time could be highly reduced but it led to lower accuracy. The average accuracy is calculated as $89.51 \%$.

Next, the delay associated with the detection of the onset is also crucial and with this methodology, since the data being used was 400 seconds prior to the seizure onset, with the high end faster processing unit, it could be done within the same seconds of delay.

\section{CONCLUSION}

This paper discusses the multi-resolution analysis of the EEG signal under different bands for a specific patient prior to the occurrence of the epilepsy. The EEG signal is used as an important tool to inspect the cases of epileptic seizures. This research work intended to automate the process and perform the analysis of those signal frequency domain in order to extract the significant features out of it. Those features were used as important parameters deciding the occurrence of epilepsy.

The observation of 41 different cases of the patient has shown a unique pattern for the epileptic case and non-occurrence for the non-epileptic session with the PPV, NPV and accuracy as discussed in [27]. Similar unique pattern weren't observed in other patient. Hence based on the analysis of the first patient, similar region is used for other patients and PCA and NN were implemented. It had resulted in $100 \%$ accuracy for NN only however with large amount of data. With PCA the data input is reduced but has resulted in lower accuracy level. Since, the prediction of the occurrence of the epileptic seizure is more crucial to alert the patient or his/her near ones, this finding could be a boon for such and thus can be used as a very useful feature for the development of the prediction model for the detection of the onset of the epileptic seizure and alerting mechanism.

\section{Acknowledgment}

The authors would like to thank the University Grants Commission (UGC) of Nepal for funding this project [30]. Also, the authors are very grateful to the MIT BIH for availing the database of epileptic seizure for free for research purpose.

\section{References}

[1] Niedermeyer E. and da Silva F.L. (2004). Electroencephalography: Basic Principles, Clinical Applications, and Related Fields, Lippincot Williams \& Wilkins. ISBN 0-7817-5126-8

[2] S. Tong and N.V.Thacor, Engineering in Medicine \& Biology- Quantitative EEG Analysis Methods and Clinical Applications, Boston/London: Artech House, 2009. 
[3] Thurman, DJ; Beghi, E; Begley, CE; Berg, AT; Buchhalter, JR; Ding, D; Hesdorffer, DC; Hauser, WA; Kazis, L; Kobau, R; Kroner, B; Labiner, D; Liow, K; Logroscino, G; Medina, MT; Newton, CR; Parko, K; Paschal, A; Preux, PM; Sander, JW; Selassie, A; Theodore, W; Tomson, T; Wiebe, S; ILAE Commission on, Epidemiology (September 2011). "Standards for epidemiologic studies and surveillance of epilepsy.".Epilepsia. 52 Suppl 7: 2-26.

[4] "Epilepsy". Fact Sheets. World Health Organization. October 2012. Retrieved January 24, 2013.

[5] Brodie, MJ; Elder,AT, Kwan, P(November 2009). "Epilepsy in later life". Lancet neurology 8 (11): 1019-30.

[6] Wyllie's treatment of epilepsy: principles and practice. (5th ed.). Philadelphia: Wolters Kluwer/ Lippincott Williams \& Wilkins. 2010. ISBN 9781-58255-937-7.

[7] Newton, CR (29 September 2012). "Epilepsy in poor regions of the world.". Lancet 380 (9848): 1193-201.

[8] Wilden, JA; Cohen-Gadol, AA (15 August 2012). "Evaluation of first nonfebrile seizures.". American family physician 86 (4): 334-40.

[9] Berg, AT (2008). "Risk of recurrence after a first unprovoked seizure". Epilepsia. 49 Suppl 1: 13-8.

[10] L Devlin, A; Odell, M; L Charlton, J. Koppel, S (December 2012). "Epilepsy and driving: current status of research" Epilepsy research 102 (3): 135-52.

[11] J. Gotman, Automatic recognition of epileptic seizure in EEG, Electroencephalograpy and Clinical Neurophysiology, 54:530-540, 1982.

[12] Hao QU and Jean Gotman, Improvement in seizure detection performance by automatic adaptation to the EEG of each patient, Electroencephalography and Clinical Neurophysiology, 86:79-87, 1993.
[13] Scott Wilson, Mark Scheuer, Ronald Emerson, and Andrew Gabor, Seizure detection: Evaluation of the reveal algorithm, Clinical Neurophysiology, 10:2280-2291, 2004.

[14] M.E. Saab and J. Gotman, A system to detect the onset of epileptic seizures in scalp EEG, Clinical Neurophysiology, 116: 427-442, 2005.

[15] Hao Qu, Self adapting algorithms for seizure detection during EEG monitoring, PhD dissertation, McGill University, 1995.

[16] Hao $\mathrm{Qu}$ and Jean Gotman, Improvement in seizure detection performance by automatic adaptation to the EEG of each patient, Electroencephalography and clinical neurophysiology, 86:79-87, 1993.

[17] Hao Qu and Jean Gotman, A seizure warning system for long term epilepsy monitoring, Electroencephalography and clinical neurophysiology, 45:2250-2254, 1995.

[18] Hao Qu and Jean Gotman, A patient specific algorithm for the detection of seizure onset in long term EEG monitoring, IEEE transactions on biomedical engineering, 44:115-122, 1997.

[19] Ali Shoeb, Application of Machine Learning to Epileptic Seizure Onset Detection and Treatment, $\mathrm{PhD}$ Thesis, Massachusetts Institute of Technology, September 2009.

[20] Sriram Ramgopal et al, Seizure detection, seizure prediction, and closed-loop warning systems in epilepsy, Epilepsy \& Behavior, 37 (2014) 291307, 2010, Online: https://www.sciencedirect. com/science /article/pii/S1525505014002297, access date: May 2018.

[21] Nhan Duy Truong, Levin Kuhlmann, Mohammad Reza Bonyadi, Jiawei Yang, Andrew Faulks, Omid Kavehei, Supervised learning in automatic channel selection for epileptic seizure detection, Expert systems with application, Volume 86, 15 November 2017, Pages 199-207, Online: https:// www.sciencedirect.com/science/article/pii/S09 57417417303779, accessed date: May 2018.

[22] CHB MIT Scalp EEG database, Online: http:// physionet.org/physiobank/database/chbmit, access date: May 2018. 
[23] Ali Shoeb, John Guttag, 27th International Conference on Machine Learning (ICML), June 21-24, 2010, Haifa, Israel.

[24] Ali Shoeb, Herman Edwards, Jack Connolly, Blaise Bourgeois, S. Ted Treves, John Guttag, Patient-Specific Seizure Onset Detection, Epilepsy and Behavior. August 2004, 5(4): 483498. [doi:10.1016/j.yebeh.2004.05.005]

[25] Moller, Neural Networks, Vol. 6, 1993, pp. 525-533.

[26] Jolliffe, I. T. Principal Component Analysis. 2nd ed., Springer, 2002.

[27] Sachin Shrestha et. al, Analysis of EEG signal of specific epileptic patient prior to its occurrence, SCITECH Nepal, Vol 13, No 1, September 2018.
[28] Howard Hamilton, Confusion matrix, Online: http://www2.cs.uregina.ca/ d dbd/cs831/notes/ confusion_matrix/confusion_matrix.html, access date: December 2018.

[29] David Felson, Positive and Negative predictive values, Online: http://sphweb.bumc.bu.edu/otlt/ MPH-Modules/EP/EP713_Screening/ EP713_ Screening5.html, access date: May 2018.

[30] University Grants Commission, Online: http:// www.ugcnepal.edu.np, access date: May 2018. 